\title{
Spatial determinants of visual masking: Effects of mask size and retinal position
}

\author{
JOSEPH F. STURR, THOMAS E. FRUMKES ${ }^{1}$ AND DONNA M. VENERUSO ${ }^{2,3}$
}

SYRACUSE UNIVERSITY

\begin{abstract}
Visual masking was studied in three observers by measuring the threshold for detection of a small black disc (test target) with and without prior exposure to a concentric larger black disc (masking target). Independent variables were size of mask and retinal position. Results showed that: (1) for all retinal positions, decreasing the size of the masking target produced a greater masking effect; (2) for each size of mask, there was a greater masking effect in the periphery than in the fovea; (3) in the fovea, the largest mask produced a lowered threshold for test target detection, suggesting summation or facilitation. These results were discussed in terms of contour interaction and signal-to-noise ratio.
\end{abstract}

\section{Introduetion}

Since the classic studies of Werner (1935) and Crawford (1947), the importance of temporal factors in visual masking has been clearly demonstrated for both dark figures and light flashes (for reviews, see Raab, 1963; Sperling, 1965). To date, however, only a few investigators (Battersby \& Wagman, 1962; Kolers, 1962) have been concerned with spatial factors, and none to our knowledge have systematically investigated the effects of retinal position. The importance of these latter parameters cannot be overemphasized, since contour effects have been shown to be significant in visual masking (Werner, 1935). According to a contour theory, the greater the proximity of the masking and test targets, the greater the masking effect. On the other hand, visual masking has also been explained by signal detection theory, in which the masking target produces a background "noise" in the visual system, thus obscuring the appearance of a small test "signal." Such a theory would predict that the larger the mask, the greater the masking effect. It would appear that the neuroanatomical arrangements in the fovea (fine grain, 1:1 neural pathway) vs. the periphery (coarse grain, greater neural convergence) would provide a basis for differentiating between contour interactions vs. signal-to-noise ratio. Therefore, the purpose of this investigation was to compare masking as a function of size of mask in the fovea vs. three peripheral loci. Method

The stimuli (A in Fig. 1) were presented binocularly (either foveally or at $2^{\circ}, 5^{\circ}$, and $7^{\circ}$ in the horizontal meridian of the left visual field) by means of a Gerbrands tachistoscope (Model T-1C). The test flash was always $10^{\prime}$ of arc, and there were three sizes of masking targets-15', 22.5', and $30^{\prime}$ of arc.
The masking target and fixation crosshair were presented on one screen and the test target on another, combined by a half-silvered mirror to appear concentrically in one field. The test, masking, and fixation targets were black. The background illumination of the white adapting screen was approximately $8.0 \mathrm{ml}$ and the contrast between masking target and test screen was almost $100 \%$. The test target was presented on a grey background; thus, the contrast was much lower.

The experimental method is shown in B of Fig. 1. For determining the Resting Threshold (RT), the adapting field was on continuously except for the brief interval when the test target was presented. For the experimental condition, the adapting field and masking target were visible except when the test flash was presented. The temporal interval between the mask and test was fixed. The dependent variable was the duration of the test target for threshold detection. Although the test and masking targets were presented sequentially, they appeared to be simultaneous, as shown in Fig. 1.

The three emmetropic observers (ages 20-24) first had several practice sessions during which approximate thresholds were obtained, using a bracketing method.

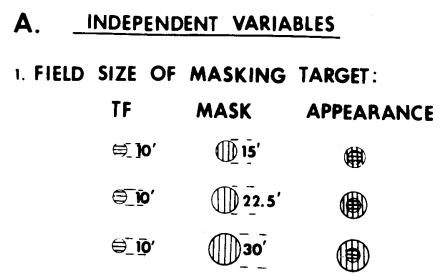

2. RETINAL POSITION: $0^{\circ}$ (fovea) $2^{\circ}, 5^{\circ}, 7^{\circ}$

B. EXPERIMENTAL PROCEDURE

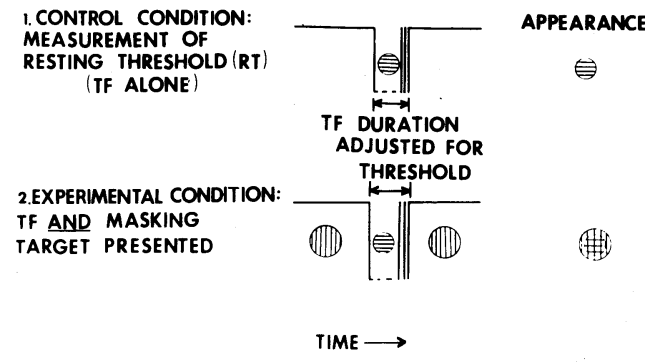

Fig. 1. Independent variables and experimental procedure. $\mathbf{T F}=$ test flash. 


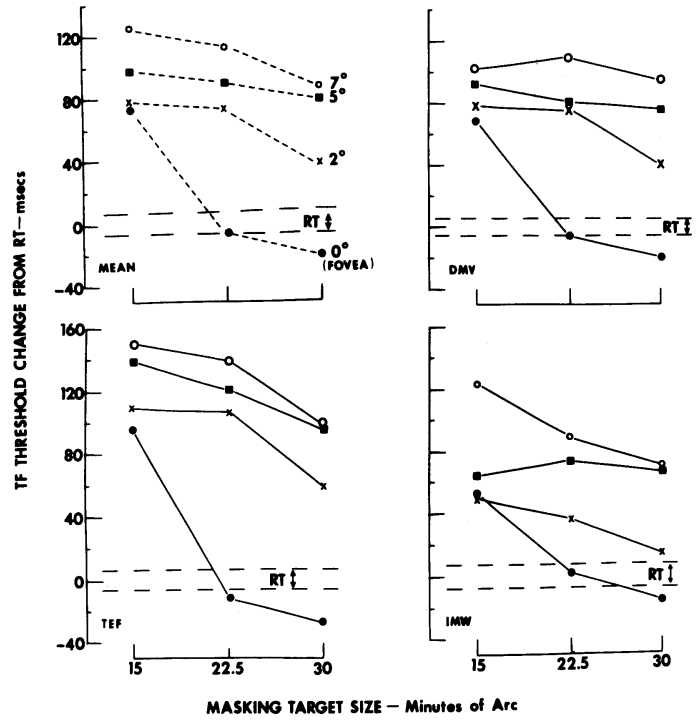

Fig. 2. Masking functions for three observers (solid line functions) and mean data (dotted line functions). Retinal positions for individual observers as indicated on mean data.

All subsequent data were collected using the method of limits. At least two separate testing sessions were recorded for each $\mathrm{S}$ in each condition, with more trials for the conditions with higher variability. Each trial consisted of three alternating descending and ascending series, blanks being shown at request and at irregular intervals. There was a variable 5 to $10 \mathrm{sec}$. interval between stimulus presentations.

\section{Results}

Figure 2 illustrates individual data from three observers and the mean data (dotted functions). For each function, change from resting threshold (RT) is plotted on the ordinate, masking target size on the abscissa. Each symbol represents a different retinal position. The horizontal dash lines above and below zero on the ordinate represent the mean deviation in the resting threshold. The average variability of the other data points was $\pm 12 \mathrm{msec}$.

In general, the curves from all three observers show similar trends, and the mean functions are representative of the individual data. For all four retinal positions, decreasing masking effects are shown with larger masking targets: that is, the test target is most difficult to detect when paired with the smallest mask. For each masking target size, there is a greater masking effect when more peripheral retinal regions are stimulated, but the slope of the masking function is much more steep for the foveal data than for the three peripheral conditions. Finally, for the largest mask, the foveal data fall below zero, suggesting a summation or facilitation effect.

\section{Diseussion}

The present findings, showing decreased masking effects with increasing size of mask are in agreement with the data of Battersby \& Wagman (1962) and Kolers (1962), extending their results to different retinal positions. In general, the results favor an interpretation based upon competing contours. Since the slopes of the masking functions become less steep in the periphery, contour effects appear to play a more critical role in the fovea for the parameters of the targets used in the present study. A plausible explanation for this is indicated by the anatomical arrangement of the retina. That is, as the receptor cell density decreases in the periphery, with greater neural convergence, the nervous system cannot make efficient use of the information provided by differences in contour, and perhaps functions more on the basis of signal-to-noise differences. This idea will be tested in future experiments by stimulating regions farther in the periphery where even greater neural convergence is known to occur. If signal detection is the underlying mechanism, then one would expect increasing masking effects with increasing field size.

The most surprising finding of the present study was facilitation in the fovea with the largest mask. More detailed studies of this facilitatory effect are currently under investigation.

\section{References}

Battersby, W. S., \& Wagman, I. H. Neural limitations of visual excitability. IV: Spatial determinants of retrochiasmal interaction. Amer. J. Physiol., 1962, 203, 359-365.

Crawford, B. H. Visual adaptation in relation to brief conditioning stimuli. Proc. Roy. Soc. B., 1947, 134, 283-302.

Kolers, P. A. Intensity and contour effects in visual masking. Vis. Res., 1962, 2, 277-294.

Raab, D. H. Backward masking. Psychol. Bull., 1963, 60, 118-129. Sperling, G. Temporal and spatial visual masking. I. Masking by impulse flashes. J. Opt. Soc. Amer., 1965, 55, 541-559.

Werner, H. Studies in contour: I. Qualitative analyses. Amer. $J$. Psychol., 1935, 47, 40-64.

\section{Notes}

1. U. S. P. H. Predoctoral Trainee in Physiological Psychology. 2. Participant in N. S. F. Undergraduate Science Education Program.

3. The authors wish to thank Mr. Ivan M. Weis for serving as an observer. 